\title{
Effective colon cancer prophylaxis in mice using embryonic stem cells and carbon nanotubes
}

\author{
Teodora Mocan' \\ Cornel lancu ${ }^{2}$ \\ 'Department of Physiology, \\ ${ }^{2}$ Third Surgery Clinic, Department \\ of Nanomedicine, luliu Hatieganu \\ University of Medicine and \\ Pharmacy, Cluj-Napoca, Romania
}

This article was published in the following Dove Press journal:

International Journal of Nanomedicine

9 September 2011

Number of times this article has been viewed
Introduction: In recent years, a new concept of an anticancer vaccine has been proposed to prevent and control the proliferation and expansion of cancer cells by eliciting an immune boost in biological systems. The recent literature supports the role of embryonic stem cells (ESC) as cellular agents that stimulate the biological systems to destroy cancer cells. However, at present, a true anticancer vaccine remains elusive. There are several lines of evidence showing that carbon nanotubes may be used to initiate and maintain immune responses.

Objective: The authors proposed to test the therapeutic potential of multiwalled carbon nanotubes (MWCNTs) combined with ESC as agents to induce an immune boost and provide subsequent anticancer protection in mice.

Methods: C57 BL/6 mice were immunized with ESC and MWCNTs.

Results: The proposed vaccine led to significant antitumor responses and enhanced tumor rejection in mice with subcutaneous inoculation of MC38 colon malign cells compared with groups only administered ESC, only MWCNTs, and controls.

Conclusion: The application and potential of ESC combined with MWCNTs as anticancer immunization agents may represent the beginning of a new chapter in the treatment of colon cancer.

Keywords: carbon nanotubes, embryonic stem cells, synergistic enhancement, immunization, colon cancer, vaccine

\section{Introduction}

Colon cancer is a major cause of mortality worldwide, and this is expected to rise in the coming years. ${ }^{1}$ Current therapeutic strategies in colon cancer include surgical resection of the primary tumor, chemotherapeutic drugs, and radiotherapy. ${ }^{2}$ The development of nanoscale drug delivery systems represents an exciting and new approach to cancer treatment. ${ }^{3}$ The ultimate aim when using nanoscale drug systems is delivery of high doses of active bionanomolecules at specific sites while simultaneously reducing systemic toxicity. ${ }^{4}$ Very recent clinical trials suggest that nanoscale drug delivery systems, such as doxorubicin encapsulated in liposomes $\left(\text { Doxil }^{\circledR}\right)^{5}$ and paclitaxel attached to nanoparticles (Abraxane $\left.{ }^{\mathbb{R}}\right),{ }^{6}$ could prolong survival in advanced cancer. One remarkable property of these nanosystems is their ability to activate the immune system, which could form an attractive basis for development of a cancer vaccine. ${ }^{7}$ Although such drug delivery systems hold tremendous potential for the future prevention of cancer, a true anticancer vaccine remains elusive. ${ }^{8}$ Nanotechnology has already shown promising results in the field of anticancer vaccines. For instance, inert nanobeads, recombinant virus-like particles, and immunostimulating complexes, are being used in cancer vaccine research due to their efficacy at eliciting both cellular and humoral immune responses. ${ }^{9}$
Correspondence: Teodora Mocan and Cornel lancu

University of Medicine and Pharmacy,

Victor Babes, 8 Cluj-Napoca, Romania

Tel +40264439696

Fax +40 264439696

Email teodora.mocan@umfcluj.ro; cornel. iancu@umfcluj.ro submit your manuscript $\mid$ www.dovepress.com

Dovepress

http://dx.doi.org// 0.21 47//JN.S24060 
During the last decade, advances in functionalization chemistry have been one of the driving forces in the development of new classes of allotropes of carbon for applications in biology and medicine. ${ }^{10-14}$ Due to their unique physical and chemical properties, carbon nanotubes hold great promise for drug delivery and cancer therapy. ${ }^{15}$ There are encouraging data suggesting that carbon nanotubes may be used to initiate and maintain immune responses. ${ }^{16} \mathrm{When}$ bound to tumor antigens, carbon nanotubes elicit a specific antitumor response in animal models. ${ }^{17}$ Furthermore, it has been shown that peptide-functionalized carbon nanotubes can act as proficient immunomodulators and consequently generate specific antibody responses. ${ }^{18}$ Moreover, ex vivo clonal expansion of $\mathrm{T}$ cells with antibodylinked carbon nanotubes results in $\mathrm{T}$ cell activation and might lead to the development of novel immunotherapies. ${ }^{19}$

It has been shown in animal models that significant lymphocyte proliferation and secretion of cytokines may help to rebuild host immunity against cancer and consequently generate obvious antitumor immunity. Despite their proven role in proliferation of $\mathrm{T}$ lymphocytes, especially the proliferation of CD8+ (cytotoxic T) lymphocytes, ${ }^{19}$ which are the main antitumor effector cells, ${ }^{20,21}$ there are currently no studies that explore the concept of cancer prophylaxis mediated by carbon nanotubes.

Recently published data support the role of embryonic stem cells (ESC) as a cellular cancer vaccine that stimulates biological systems to destroy colon cancer cells by eliciting an immune boost. ${ }^{22}$ This implication is supported by the fact that ESC prevent and control proliferation and expansion of malignant tumors in vivo by formation and development of CD4+ and CD8+ T lymphocytes. ${ }^{23}$

Considering all these data together, we hypothesized that combined administration of both ESC and carbon nanotubes would function as a powerful nanobiosystem to induce and rebuild antitumor immunity in colon tumor animal models. Here we demonstrate that simultaneous immunization with ESC and MWCNT leads to significant antitumor responses and enhanced tumor rejection in mice with subcutaneous inoculation of malignant colon cells. The application and potential of ESC and MWCNT as anticancer vaccines may represent the beginning of a new chapter in the treatment of colon cancer.

\section{Materials and methods}

\section{Carbon nanotubes}

MWCNT self-assembled into bundles ( $>99 \%$ carbon basis, $8-15 \mathrm{~nm}$ [outside diameter] $\times 3-5 \mathrm{~nm}$ [inside diameter] $\times 10-50 \mu \mathrm{m}$ [length]) were purchased from Sigma Chemical Company, Deisenhofen, Germany (Figure 1A).

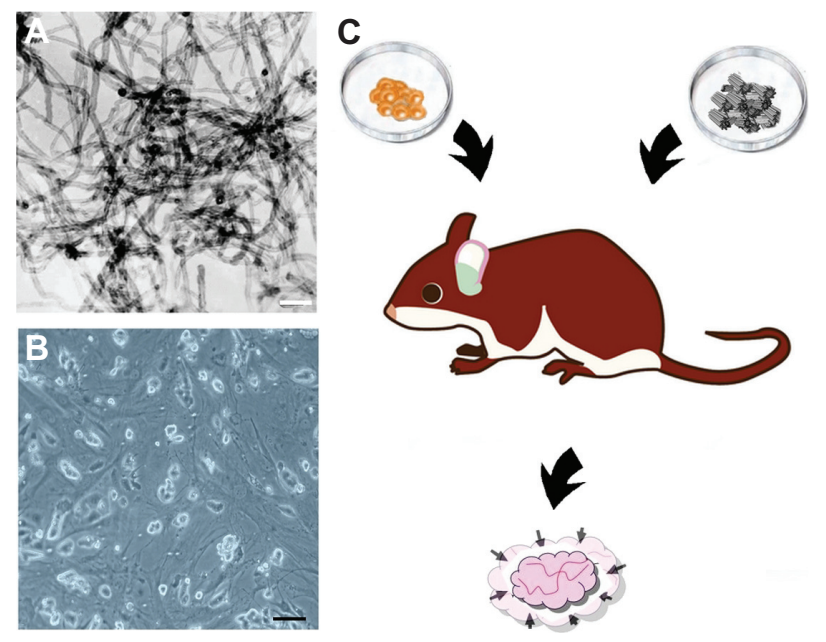

Figure I (A) Transmission electron micrographs of MWCNTs used for the immunization. The scale bar represents $100 \mathrm{~nm}$. (B) In vitro C57 BL/6 mouse embryonic stem cells used for immunization (scale bar: $50 \mu \mathrm{m}$ ). (C) Schematic illustration of the proposed vaccine.

Abbreviation: MWCNT, multiwalled carbon nanotube.

The nonconjugated highly purified MWCNT control solution was prepared as previously described. ${ }^{24}$ The product obtained was diluted in physiological saline solution $(\mathrm{NaCl} 0.9 \%)$ at a $1: 10(\mathrm{v} / \mathrm{v})$ ratio.

\section{Cell lines}

C57 BL/6 mouse ESC were purchased from the American Tissue Culture Collection (ATCC), and cultured in mouse ESC growth medium on plates precoated with gelatin solution and C57 BL/6 mouse embryonic fibroblasts (ATCC) as feeder cells (Figure 1B). For use as cellular therapy, ESC were centrifuged (1000 rpm/10 minutes) and the supernatant discarded. The viability of ESC cells was assessed using the $0.4 \%$ Trypan blue dye method prior to administration. Cell concentrations were adjusted to $1 \times 105$ cells $/ \mu \mathrm{L}$.

\section{Mice}

Six-week-old C57 BL/6 female mice weighing 20-25 g were purchased from Jackson Laboratory (Bar Harbor, ME) and housed under sterile conditions in laminar air flow cabinets in accordance with standard guidelines. All animal experiments were approved by the Institutional Ethics Committee.

\section{Vaccine administration}

Forty $\mathrm{C} 57 \mathrm{BL} / 6$ mice were randomly divided into four groups. The first group received $1 \times 10^{6} \mathrm{C} 57 \mathrm{BL} / 6 \mathrm{ESC}$ (inoculated subcutaneously) weekly for 6 weeks; the second group received $300 \mu \mathrm{L}$ of about $0.1 \mathrm{mg} / \mathrm{mL}$ MWCNT intravenously administered into the tail vein for 6 weeks (at 1-week intervals); the third group received both C57 BL/6 
ESC and MWCNT at the same concentrations $\left(1 \times 10^{6} \mathrm{C} 57\right.$ $\mathrm{BL} / 6 \mathrm{ESC}+300 \mu \mathrm{L}$ of approximately $0.1 \mathrm{mg} / \mathrm{mL}$ MWCNT, injected separately, at the same administration intervals, ie, weekly for 6 weeks); and the control group received $1 \mathrm{~mL}$ of physiological saline solution in the tail vein in accordance with the above administration schedule.

\section{MC38 murine colon cancer xenografts}

After 6 weeks of administration (physiological saline solution, ESC, MWCNT, or ESC + MWCNT), MC38 (ATCC) colon cancer cells were injected subcutaneously into the mice, according to a previously described method. ${ }^{22,23,25}$ Subcutaneous tumor size was measured every 3 days using digital vernier calipers. Tumor volume was calculated based on three axis measurements. ${ }^{22}$

\section{Lymphocyte counts}

At the end of immunization (physiological saline solution, ESC, MWCNT, or ESC + MWCNT), citrate-anticoagulated blood was acquired from the venous sinus of the eye orbit ${ }^{26}$ in mice from all groups. Sampled blood was stained with antibodies for CD4+ and CD8+ (BD Bioscience Pharmingen, San Diego, CA) for sorting T lymphocytes by a Beckman CellLAb Quanta flow cytometer (Beckman Coulter, Indianapolis, IN). ${ }^{23}$

\section{Antigen-specific T cell response}

Spleens were aseptically removed from selected sacrificed animals in the treatment and control groups at the end of the study. Each spleen was cut into small pieces in RPMI1640 medium (Sigma, St Louis, MO) and rinsed through 40 mesh stainless steel screens. Cell suspensions were further purified by Ficoll-Hypaque gradient centrifugation.

The frequencies of interferon-gamma (IFN- $\gamma$ ) and tumor necrosis factor (TNF)-secreting CD8 T lymphocytes in a splenocyte population were determined by intracellular cytokine staining. Cells were fixed in $2 \%$ paraformaldehyde, permeabilized with PermWash buffer (Pharmingen), and incubated with anti-IFN- $\gamma$ allophycocyanin antibody (BD Biosciences) and anti-TNF-phycoerythrin antibody (Abcam, Cambridge, UK). Samples were acquired on a Beckman CellLAb Quanta flow cytometer, and the data were analyzed using CellQuest software (Becton Dickinson Immunocytometry Systems, San Jose, CA).

\section{Immunohistochemistry analysis}

On day 21 following subcutaneous inoculation of MC38 colon cancer cells (this was considered the endpoint of the study) the mice were sacrificed. Tumors and spleens were excised and immersed in $4 \%$ formaldehyde solution. The tissues were embedded in paraffin wax and serially sectioned. Tissue sections were deparaffinized and rehydrated in a graded ethanol series followed by incubation with $3 \%$ hydrogen peroxide for 15 minutes, and 15-minute rinses in three changes of phosphate-buffered saline. Next, in order to suppress nonspecific background staining, the sections were blocked with normal goat serum. The diluted (1:100) rabbit antimouse CD4 and CD8 antibodies (Sigma) were applied to the sections at $4{ }^{\circ} \mathrm{C}$ for 12 hours. Quantification of CD4+ or CD8+ lymphocytes was performed using the mean optical density method as described elsewhere. ${ }^{27}$ The sections were then incubated with biotinylated goat antirabbit IgG for 30 minutes at room temperature and processed according to the manufacturer's protocol. Specific visualization of tissues was performed using an Olympus BX60 light microscope equipped with a camera system (Hamburg, Germany).

\section{TUNEL assay}

The sections harvested from tumors at the endpoint (on day 21 following subcutaneous inoculation of colon cancer cells) were incubated with protease $\mathrm{K}$ for 30 minutes at room temperature for antigen retrieval and further prepared according to the TUNEL TMR red apoptosis detection kit (Roche Applied Science, Branford, CT). The red-labeled TUNEL-positive cells were visualized under a fluorescent microscope (LP585 emission). Quantification of positively stained nuclei was performed using the mean optical density method.

\section{Cytokine expression}

Fresh blood from all mice was obtained at the same time and in the same way as previously described, and serum was collected. Quantification of interleukin (IL)-10 IL-2, and IFN- $\gamma$ was performed using commercially available enzyme-linked immunosorbent assay kits (Sigma) in accordance with the manufacturer's instructions and analyzed on an enzyme-linked immunosorbent assay reader (Labsystem Multiskan Plus, Helsinki, Finland). Minimum detectable cytokine concentrations for these assays as provided by the manufacturer were IL-2 (4 pg/mL), IL-10 (2 pg/mL), and IFN- $\gamma(4 \mathrm{pg} / \mathrm{mL})$.

\section{Electron microscopy imaging}

The ultrastructural morphology of MWCNT was evaluated using a Jeol JEM 1010 transmission electron microscope (Jeol, Tokyo, Japan) as previously described. ${ }^{28}$ The images were captured using a Mega VIEW III camera (Olympus, Soft Imaging System, Münster, Germany). 


\section{Statistical analysis}

All data were expressed as means \pm standard error of the mean. Continuous data were tested for normality (KolmogorovSmirnov test) before hypothesis testing. Longitudinal data, representing repetitive measurements of the same parameter during the follow-up interval, were analyzed by construction of a kinetic curve along with area under the curve calculus. Consecutively, kinetic curves of two different groups were compared using the Mann-Whitney U test. Fisher's Exact test was used for qualitative data correlations. For all tests, a 0.05 threshold was selected for statistical significance. Statistical data analysis was performed using SPSS 17.0 software (SPSS Inc, Chicago, IL).

\section{Results and discussion}

The first in vitro published observation of the potential of embryonic materials as a vaccine to prevent the development of tumor xenografts in animal models ${ }^{29}$ stimulated significant interest and research, leading to rapid development in this field. Preliminary data support the role of ESC as effective cellular agents that reverse the immune dysfunction that causes cancer and induces antitumor immunity in tumorloading mice of different stages. ${ }^{23}$ On the other hand, several authors have suggested that carbon nanotubes have impressive immunomodulatory properties and are shown to enhance both the kinetics and magnitude of $\mathrm{T}$ cell and macrophage activation. ${ }^{19}$ It is well known that one of the reasons for failure of the immune system to eliminate a tumor mass is lack of appropriate activation of the lymphocyte population to a threshold required for tumor rejection. ${ }^{30}$ Given these data and the described role of MWCNT in proliferation of lymphocytes, we reasoned that administration of both carbon nanotubes and $\mathrm{ESC}$ would provide a better immune response and stronger antitumor immunity (Figure 1C).

\section{Outcome and biodistribution after administration of MWCNT}

Neither mortality nor significant loss of body weight was observed in any of the mice among the four study groups during the 9 -week period of the study. These findings are similar to other reports showing $100 \%$ long-term survival in mice following MWCNT administration at similar doses. ${ }^{31}$ Following administration, MWCNT deposits were found in the liver, spleen, kidney, and lungs, but not in tumor tissue.

Despite their unique features, the toxicity and biological interactions of carbon nanotubes represent a major concern, with several authors pointing to their similarity to asbestos fibers. ${ }^{32}$ Many factors attributed to carbon nanotubes, such as biofunctionalization, length, concentration, duration of exposure, and methods of dispersion, have been associated with carbon nanotube toxicity in both in vitro and in vivo studies. $^{33}$

To date, few in vitro experiments assert that oxidative stress from carbon nanotubes is a major result of toxicity. However, most of the studies suggesting that carbon nanotubes are nontoxic in vivo outnumber those proposing otherwise. It has been stated that the toxicity of carbon nanotubes is negligible in mice with chronic exposure to carbon nanotubes. ${ }^{16}$

\section{Tumor development}

Following administration of the proposed vaccines, the kinetics of tumor growth in the MC38 colon cancer model were closely monitored (Figure 2A and C). Importantly, following treatment, volumetric evolution of the tumors (Figure 2A) revealed that, as compared with ESC, MWCNT, and the control group, the area under the curve corresponding to the ESC + MWCNT group was significantly lower $(\mathrm{ESC}+\mathrm{MWCNT}$ versus ESC, $P=0.021 ; \mathrm{ESC}+\mathrm{MWCNT}$ versus MWCNT, $P=0.014$ ). Moreover, in the ESC and MWCNT groups, the tumor growth rate was also significantly slower compared with the control group (ESC versus physiological saline solution, $P=0.042$; MWCNT versus physiological saline solution, $P=0.048$ ). This finding suggests a strong and stable antitumor response in mice using ESC, MWCNT, or ESC + MWCNT. However, the most significant tumor rejection was found in the ESC + MWCNT group, and was significantly higher than that in the other groups. We further compared the weights of tumors harvested on day 21 following administration of therapy and induction of carcinogenesis. Similarly, we observed that tumor weights were significantly lower for the ESC + MWCNT group compared with the control, ESC, and MWCNT groups (Figure 2B). Moreover, clinical expression (when subcutaneous tumors became palpable and measurable) of the colon cancer xenografts was also significantly delayed in the ESC + MWCNT group (Figure 2C). These results show that mice treated with ESC + MWCNT acquired significant antitumor immunity and prevented tumor masses from propagating and developing compared with the other groups.

\section{Proliferation of lymphocytes in peripheral blood of mice}

We next investigated the proliferation of both $\mathrm{T}$ and $\mathrm{B}$ lymphocytes in the peripheral circulation of all groups 


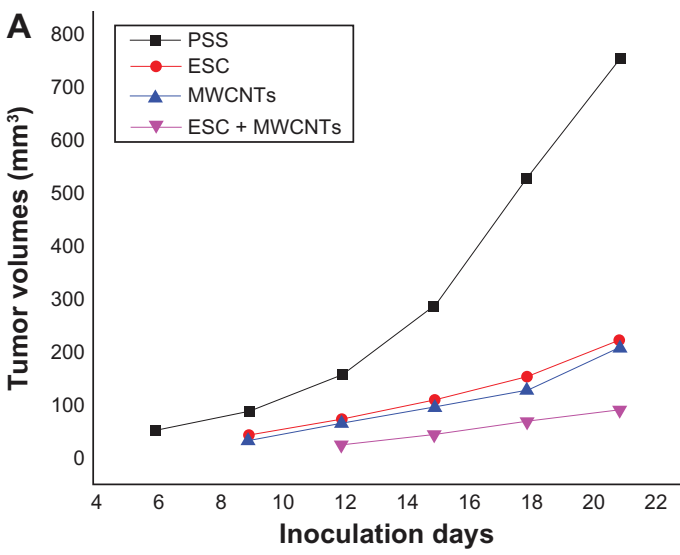

C

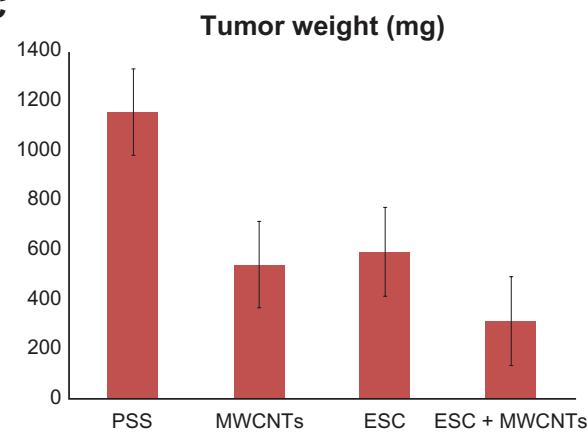

B

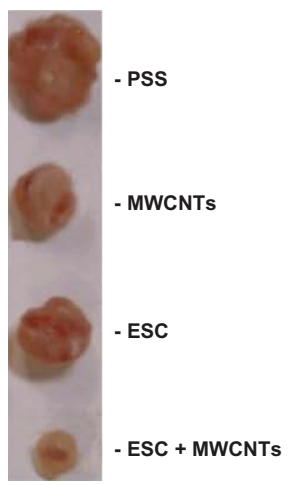

D

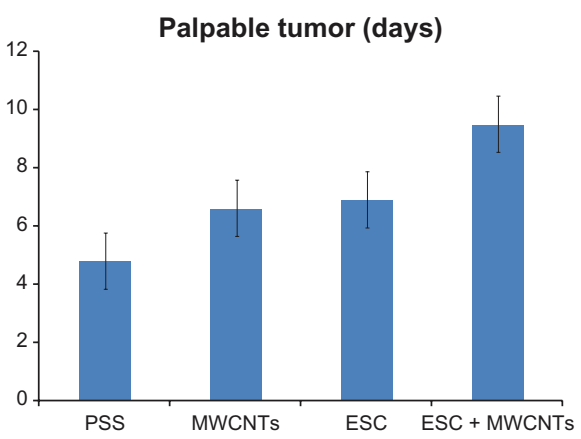

Figure 2 (A) Dynamic evolution tumor volumes following MC38 cancer cells inoculation in immunized mice. (B) Macroscopic aspect of the harvested tumors at the endpoint. (C) The results of the tumor weights at the end of the study. (D) First clinical appearance of the tumor mass following MC38 inoculation in immunized mice. Abbreviations: ESC, embryonic stem cells; PSS, physiological saline solution; MWCNT, multiwalled carbon nanotube.

of mice (Figure 3A). Notably, the absolute numbers and percentages of CD4+ and CD8+ cells in the ESC, MWCNT, and ESC + MWCNT groups were significantly higher compared with the control group ( $P<0.05$ in all cases). We observed almost similar levels of CD4+ and CD8+ T cells in the ESC and MWCNT groups (Figure 3A). Importantly, the proliferation of both $\mathrm{CD} 4+$ and $\mathrm{CD} 8+$ in the ESC + MWCNT group was significantly higher than in the ESC and MWCNT groups $(P<0.05)$. Therefore, both CD4+ and CD8+ T cells were induced in response to $\mathrm{ESC}+\mathrm{MWCNT}$ vaccination. Consequently, antitumor immunity was generated. ${ }^{34}$ We also examined the ratio of CD4+/CD8+ lymphocytes. As shown in Figure 3B, there were more cytotoxic lymphocytes (CD8+) generated in the MWCNT + ESC group, suggested by a lower ratio of $\mathrm{CD} 4+/ \mathrm{CD} 8+(P<0.05)$ compared with the other groups. This finding is of importance because CD8+ T cells are potent mediators of antitumor immunity. ${ }^{35,36}$ Moreover CD4+ T cells are also important in antitumoral immunity because, in their absence, CD8+ T cells can be depleted or lose their immunogenic capacity. ${ }^{36,37}$ Therefore, cell therapies that rely on $\mathrm{CD} 4+\mathrm{T}$ cells are far superior to therapies that only use CD8+ $\mathrm{T}$ cells. ${ }^{38}$ In the present study, following combined administration of MWCNT and ESC, we have obtained increased proliferation of both CD4+ and CD8+ lymphocytes, which constitutes the main part of antitumor effector cells. ${ }^{23,39-41}$

\section{Proliferation of lymphocytes in spleen of mice}

Figure 4 shows the proliferation of $\mathrm{CD} 4+$ and CD8+ immunostained lymphocytes in splenic tissue harvested at the endpoint. It is evident that larger numbers of CD4+ and CD8+ cells were found in the ESC + MWCNT vaccine groups compared with the ESC, MWCNT, and control group, and this finding was statistically significant $(P<0.05)$. This increased proliferation of lymphocytes was responsible for protection of ESC + MWCNT-vaccinated animals from tumor development. The mean optical density for CD4+ T lymphocytes was $0.09 \pm 0.018$ for the physiological saline solution group, $0.21 \pm 0.036$ for the ESC group, $0.24 \pm 0.023$ for the MWCNT group, and $0.31 \pm 0.054$ for the ESC + MWCNT group. For CD8+ $\mathrm{T}$ lymphocytes, the mean optical density was $0.12 \pm 0.028$ for the physiological saline solution group, $0.24 \pm 0.043$ for the ESC group, $0.22 \pm 0.037$ for the MWCNT group, and $0.34 \pm 0.064$ for the ESC + MWCNT group. For both CD4+ and CD8+, there was a statistically significant $(P<0.01)$ increase in proliferation of lymphocytes in the spleens of 

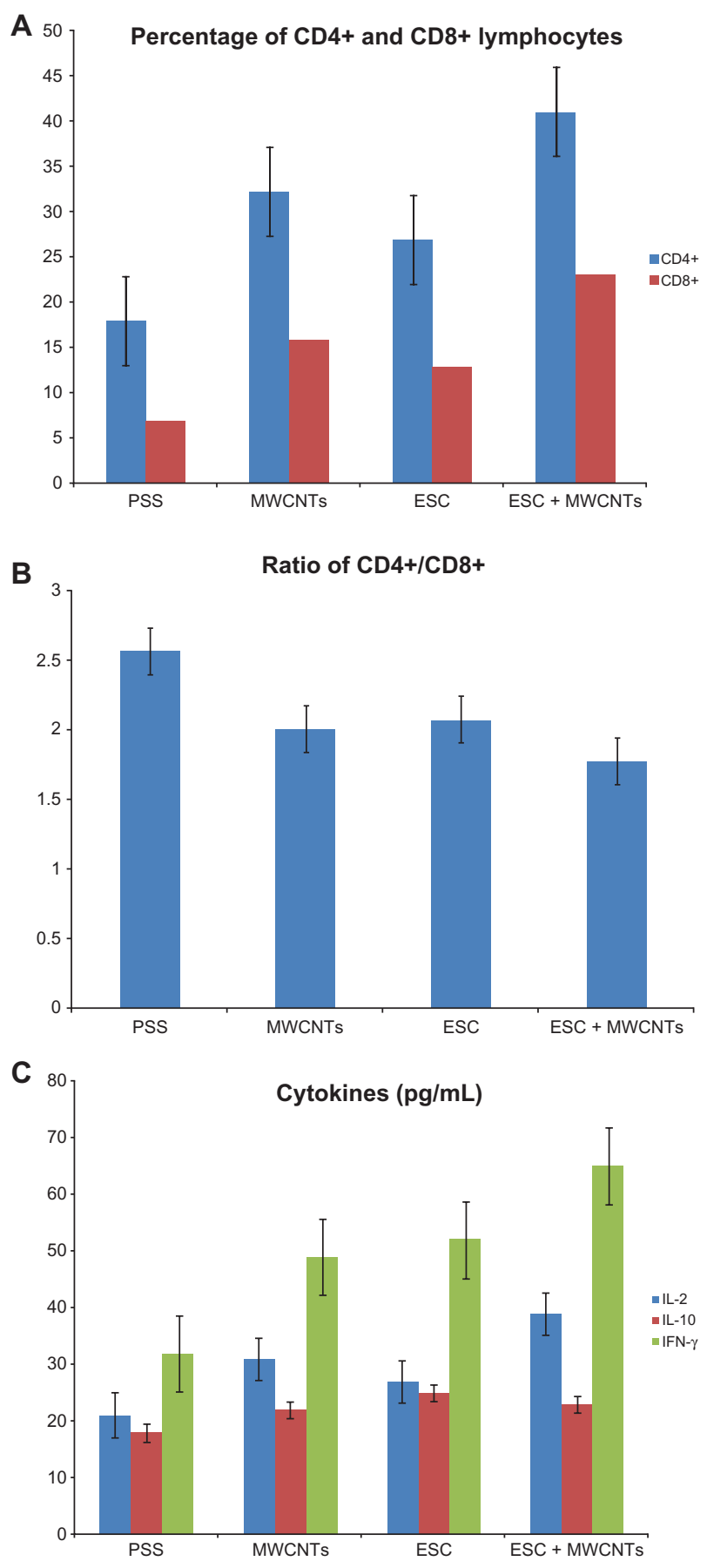

Figure 3 (A) Proliferation of CD4+ and CD8+ lymphocytes following immunization. (B) CD4+: CD8+ ratio among groups. (C) Expression of cytokines following immunization.

Abbreviations: ESC, embryonic stem cells; PSS, physiological saline solution; MWCNT, multiwalled carbon nanotube; IFN- $\gamma$, interferon $\gamma$; IL, interleukin.

mice treated with ESC + MWCNT as compared with the controls (Figure 4).

\section{Cytokine expression}

In order to shed light on the molecular mechanism involved in antitumor immunity, we next examined whether
ESC + MWCNT immunization resulted in production of antitumor-specific cytokines by lymphocytes in serum. The process of rejection of cancer cells requires $\mathrm{T}$ cell immunity. Two types of T helper cells, categorized as Th1 and Th2 on the basis of cytokine production, have been reported to play a crucial role in regulating anticancer immune responses. ${ }^{42}$ Th1 cells produce IL- 2 and IFN- $\gamma$, while Th2 cells secrete IL-4, IL-6, and IL-10. Th1 cells mediate cellular immunity, and Th2 cells support tumoral immune responses. The cytokines produced by Th1 and Th2 cell subsets are important for the function and immune response of cytotoxic $\mathrm{T}$ lymphocytes because they can regulate the differentiation of these cells. ${ }^{43-45}$ Besides the decrease in quantities of lymphocytes, the cytokines secreted by CD4+ lymphocytes in a Th1/Th2 shift state is another abnormality of lymphocyte homeostasis induced by carcinogenesis. ${ }^{46,47}$ As a result, this state is expressed by an increase in Th2 cytokines (IL-4, IL-6, IL-10) combined with a decrease in Th1 cytokines (IL-2, 3, IFN- $\gamma$ ). We analyzed the concentration of IL-10, IL-2, and IFN- $\gamma$ by enzyme-linked immunosorbent assay (Figure 3C). The results suggested that mice in the $\mathrm{ESC}+\mathrm{MWCNT}$ vaccine group had significantly increased expression of IL-2 and IFN- $\gamma(P<0.05)$. However, there was no difference in IL-10 levels between the groups. This suggests that administration of ESC + MWCNT activated secretion of Th1 cytokines, which comprises an important part of antitumor immunity. Furthermore, the statistical difference between the treatment groups ESC + MWCNT and ESC or MWCNT suggested that administration of ESC + MWCNT could stimulate the immune system more effectively to generate lymphocytes and cytokines. Due to the altered expression of proteins involved in antigen processing and presentation, tumor cells are able to avoid recognition by the immune system, and insufficient activation of antitumor immunity led to poor protective responses. ${ }^{23,48,49}$ Both an increase in lymphocytes and cytokine secretion following ESC + MWCNT were observed in our study. Thus, the proposed vaccine accomplished the reversal of the existing immune dysfunction, further stimulating the host immune protective responses. Altogether, these data indicate that tumor-specific $\mathrm{T}$ cell immunity is enhanced after ESC + MWCNT administration in C57 BL/6 mice.

\section{Molecular mechanisms of immune boost induced by MWCNT administration}

Several reports show a significant antitumor response when TNF-immunodeficient mice are treated with TNF, suggesting 

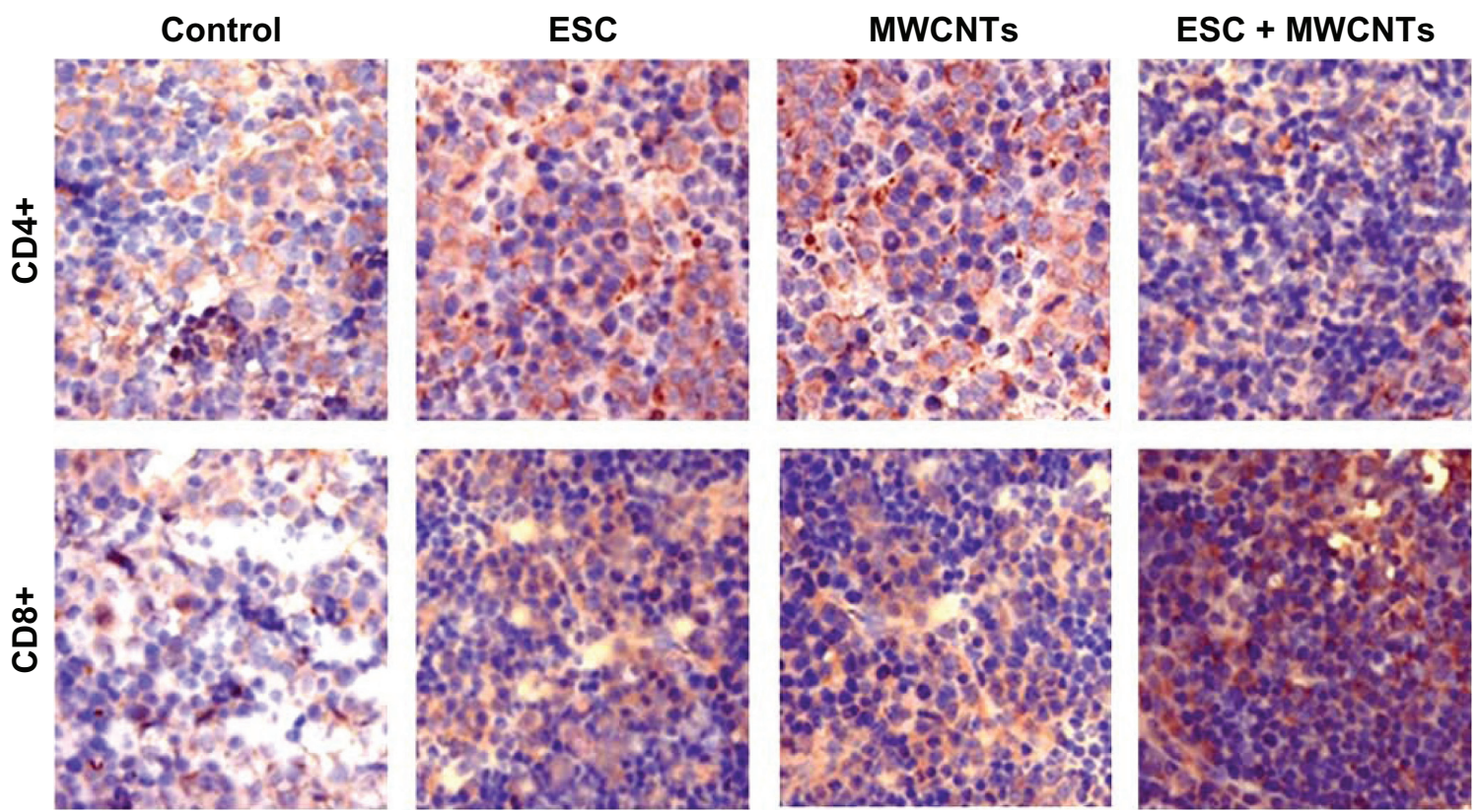

Figure 4 Proliferation of CD4+ and CD8+ lymphocytes in spleen of mice harvested at endpoint (blue).
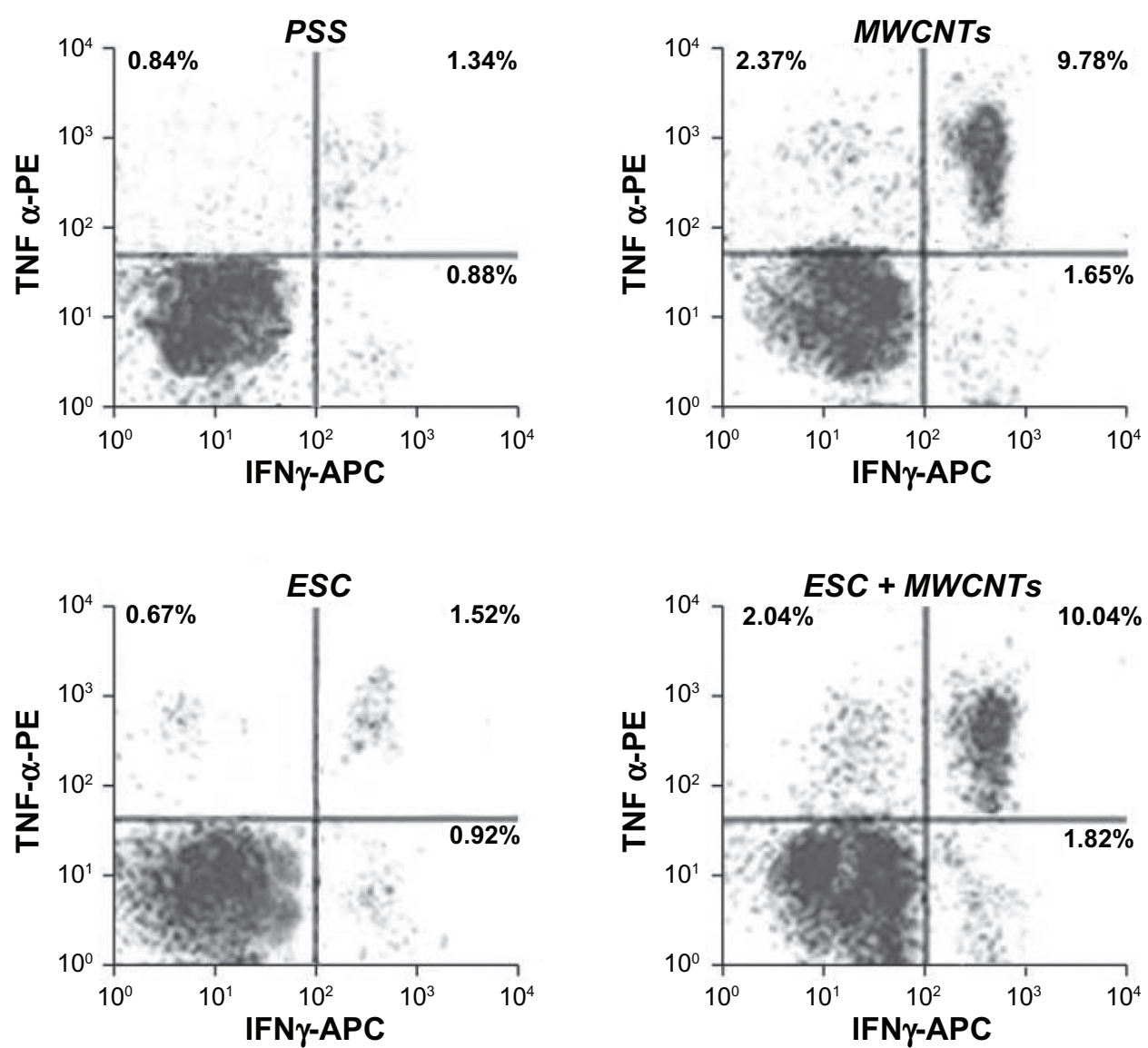

Figure 5 MWCNTs administration induces a potent specific T cell response. The left upper quadrant represents the phycoerythrin-labeled TNF-a + CD8+ T cells. The right lower quadrant represents the allophycocyanin-labeled IFN- $\gamma+C D 8+T$ cells. The cells in the upper right quadrant are TNF-a+ IFN- $\gamma+C D 8+T$ cells. The cells in the left lower quadrant represent unlabelled cells population. Cell counts are shown by dots density.

Abbreviations: MWCNT, multiwalled carbon nanotube; TNF- $\alpha$, tumoral necrosis factor; IFN- $\gamma$, interferon $\gamma$; ESC, embryonic stem cells; APC, allophycocyanin; PE, phycoerythrin. 
that TNF plays an important role in antitumor immunity. ${ }^{50}$ Most data indicate that TNF stimulates innate immunity both in vitro and in animal models and stimulates the production of other cytokines (eg, IL-1, IL-6, and IL-8) and cytotoxic agents (eg, nitric oxide and reactive oxygen species) by macrophages, which can further mediate tumor suppression. ${ }^{51}$ Alternatively, IFN- $\gamma$ enhances antitumor responses by optimizing access of antigens to HLA class $\mathrm{I}^{52}$ or by signaling through the IFN- $\gamma$ receptor on CD8+ T lymphocytes. ${ }^{53}$ In order to clarify the mechanism of the synergistic antitumor effect induced by the administration of MWCNT, we next examined IFN- $\gamma$ and TNF production in CD8+T lymphocytes harvested from splenic tissue in mice. For this purpose, IFN- $\gamma$ and TNF-secreting CD8+ T lymphocyte populations were examined in splenocytes harvested from mice at the endpoint.

As shown in Figure 5, we found that MWCNT administration in mice strongly enhanced production of IFN- $\gamma$-secreting and TNF-secreting CD8+ T cells $(P<0.001)$. In contrast, no significant difference in IFN- $\gamma$-secreting and TNF-secreting CD $8+$ production in mice vaccinated with physiological saline solution or ESC was observed. These results strongly suggest that MWCNT synergistically enhance the antitumor effect by generating IFN- $\gamma$ and TNF CD $8+$ cells.

\section{TUNEL assay}

When the mice were sacrificed, we detected cell apoptosis in tumor tissues using the TUNEL TMR kit (Figure 6). DNA disintegration as a result of cellular apoptosis may yield double-stranded and single-stranded DNA breaks (nicks). These nicks can be discovered by labeling free 3'-OH termini with modified nucleotides, such as fluorescein-dUTP, in an enzymatic reaction. This method is recognized for its high sensitivity and specificity for apoptotic cell detection in tissue sections. ${ }^{54}$ The mean optical density of red labeled apoptotic nuclei was $0.043 \pm 0.038$ for the physiological saline solution group, $0.07 \pm 0.046$ for the ESC group, $0.058 \pm 0.063$ for the MWCNT group, $0.12 \pm 0.14$ for the ESC + MWCNT group. Thus, we found that apoptosis of tumor cells in the ESC + MWCNT vaccine group was higher from that of the control group $(P<0.05)$ and the increase was marginally significant compared with the ESC or MWCNT groups ( $P=0.067$ for ESC; $P=0.072$ for MWCNT). However, no significant difference was found between the ESC and MWCNT groups. These results suggest that immunization with MWCNT and ESC could lead to significant protection against development of colon cancer. It has been shown that ESC suppress colon cancer growth by CD8+ T and CD4+ T
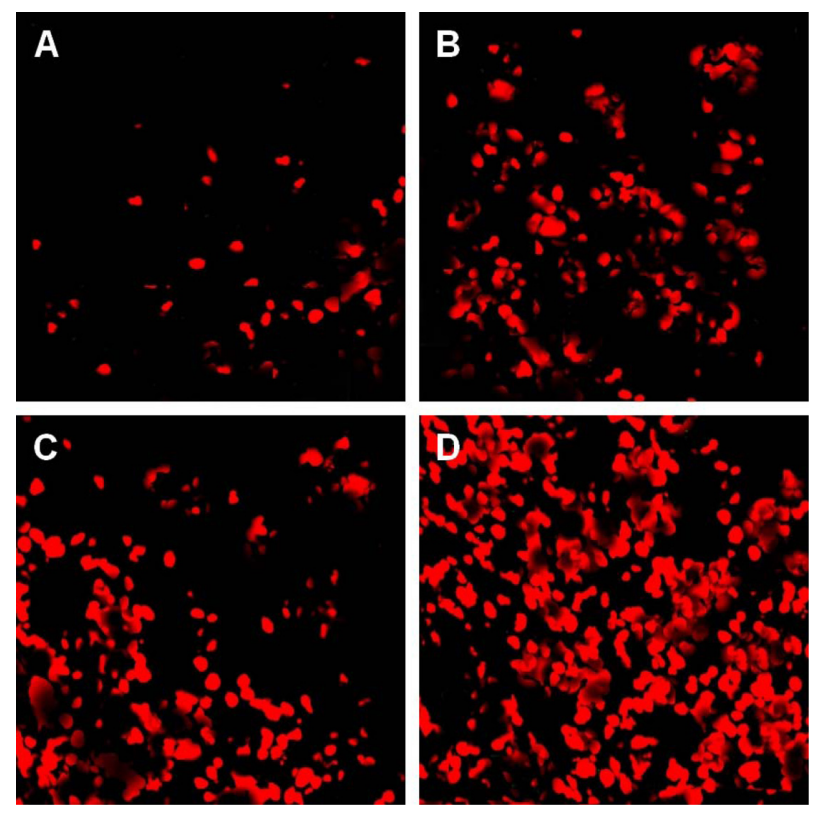

Figure 6 Cellular apoptosis in tumoral tissue harvested at the endpoint (A) control, (B) ESC, (C) MWCNTs, (D) ESC+MWCNTs.

Abbreviations: ESC, embryonic stem cells; MWCNT, multiwalled carbon nanotube.

cell activation. ${ }^{22}$ Furthermore, it has also been reported that CNTs are involved in activation of the immune response. ${ }^{19,55,56}$ In our study, we discovered a novel application of carbon nanotubes, ie, anticancer immunization. This prophylactic anticancer effect was even more pronounced when MWCNT were combined with ESC, and we showed here that systemic administration of MWCNT synergistically enhances the antitumor effect of ESC in mice.

\section{Conclusion}

The application of carbon nanotube immunotherapy represents a new paradigm in cancer treatment development. Our research could open new avenues for how cancer vaccines could be successfully developed in the future. These results are very encouraging because, for the first time, a combination of carbon nanotubes and embryonic stem cells has successfully provided activation of antitumor immunity, leading to impressive suppression of proliferation and development of malignant colon tumors. Although our approach has shown very promising results in murine models, in vivo toxicity studies of MWCNT are now required.

\section{Acknowledgment}

Financial support from the Romanian Ministry of Education and Research (PNCDI 2:VACSTEM 42-111) is gratefully acknowledged. 


\section{Disclosure}

The authors report no conflicts of interest in this work.

\section{References}

1. Jemal A, Bray F, Center MM, Ferlay J, Ward E, Forman D. Global cancer statistics. CA Cancer J Clin. 2011;61(2):69-90.

2. Segal NH, Saltz LB. Evolving treatment of advanced colon cancer. Annu Rev Med. 2009;60:207-219.

3. Subramani K, Hosseinkhani H, Khraisat A, Hosseinkhani M, Pathak Y. Targeting nanoparticles as drug delivery systems for cancer treatment. Curr Nanosci. 2009;5(2):135-140.

4. Shi J, Votruba AR, Farokhzad OC, Langer R. Nanotechnology in drug delivery and tissue engineering: From discovery to applications. Nano Lett. 2010;10(9):3223-3230.

5. ElBayoumi TA, Torchilin VP. Tumor-targeted nanomedicines: Enhanced antitumor efficacy in vivo of doxorubicin-loaded, long-circulating liposomes modified with cancer-specific monoclonal antibody. Clin Cancer Res. 2009;15(6):1973-1980.

6. Miele E, Spinelli GP, Miele E, Tomao F, Tomao S. Albumin-bound formulation of paclitaxel (Abraxane ${ }^{\circledR} \mathrm{ABI}-007$ ) in the treatment of breast cancer. Int J Nanomedicine. 2009;4:99.

7. Bachmann MF, Jennings GT. Vaccine delivery: A matter of size, geometry, kinetics and molecular patterns. Nat Rev Immunol. 2010; 10(11):787-796

8. Wang E, Monaco A, Monsurro V, et al. Antitumor vaccines, immunotherapy and the immunological constant of rejection. IDrugs. 2009; 12(5):297-301.

9. Scheerlinck JPY, Greenwood DLV. Virus-sized vaccine delivery systems. Drug Discov Today. 2008;13(19-20):882-887.

10. Xu Y, Mahmood M, Fejleh A, et al. Carbon-covered magnetic nanomaterials and their application for the thermolysis of cancer cells. Int J Nanomedicine. 2010;5:167-176.

11. Bhirde AA, Patel V, Gavard J, et al. Targeted killing of cancer cells in vivo and in vitro with EGF-directed carbon nanotube-based drug delivery. ACS Nano. 2009;3(2):307-316.

12. Khazaei A, Rad MN, Borazjani MK. Organic functionalization of singlewalled carbon nanotubes (SWCNTs) with some chemotherapeutic agents as a potential method for drug delivery. Int $J$ Nanomedicine. 2010;5:639-645.

13. Fisher JW, Sarkar S, Buchanan CF, et al. Photothermal response of human and murine cancer cells to multiwalled carbon nanotubes after laser irradiation. Cancer Res. 2010;70(23):9855-9864.

14. Wang CH, Chiou SH, Chou CP, Chen YC, Huang YJ, Peng CA. Photothermolysis of glioblastoma stem-like cells targeted by carbon nanotubes conjugated with CD133 monoclonal antibody. Nanomedicine. 2011;7(1):69-79.

15. Levi-Polyachenko NH, Merkel EJ, Jones BT, Carroll DL, Stewart JH 4th. Rapid photothermal intracellular drug delivery using multiwalled carbon nanotubes. Mol Pharm. 2009;6(4):1092-1099.

16. Kostarelos K, Bianco A, Prato M. Promises, facts and challenges for carbon nanotubes in imaging and therapeutics. Nat Nanotechnol. 2009; 4(10):627-633.

17. Meng J, Duan J, Kong H, et al. Carbon nanotubes conjugated to tumor lysate protein enhance the efficacy of an antitumor immunotherapy. Small. 2008;4(9):1364-1370.

18. Pantarotto D, Partidos CD, Hoebeke J, et al. Immunization with peptidefunctionalized carbon nanotubes enhances virus-specific neutralizing antibody responses. Chem Biol. 2003;10(10):961-966.

19. Fadel TR, Steenblock ER, Stern E, et al. Enhanced cellular activation with single walled carbon nanotube bundles presenting antibody stimuli. Nano Lett. 2008;8(7):2070-2076.

20. Begley J, Ribas A. Targeted therapies to improve tumor immunotherapy. Clin Cancer Res. 2008;14(14):4385-4391.

21. Rosenberg SA, Yang JC, Restifo NP. Cancer immunotherapy: Moving beyond current vaccines. Nat Med. 2004;10(9):909-915.
22. Li Y, Zeng H, Xu RH, Liu B, Li Z. Vaccination with human pluripotent stem cells generates a broad spectrum of immunological and clinical responses against colon cancer. Stem Cells. 2009;27(12):3103-3111.

23. Dong W, Du J, Shen H, et al. Administration of embryonic stem cells generates effective antitumor immunity in mice with minor and heavy tumor load. Cancer Immunol Immunother. 2010;59(11):1697-1705.

24. Raffa V, Ciofani G, Nitodas S, et al. Can the properties of carbon nanotubes influence their internalization by living cells? Carbon. 2008; 46(12):1600-1610.

25. Kryczek I, Wei S, Szeliga W, Vatan L, Zou W. Endogenous IL-17 contributes to reduced tumor growth and metastasis. Blood. 2009;114(2): $357-359$.

26. Fernández I, Peña A, Del Teso N, Pérez V, Rodríguez-Cuesta J. Clinical biochemistry parameters in C57BL/6 J mice after blood collection from the submandibular vein and retroorbital plexus. J Am Assoc Lab Anim Sci. 2010;49(2):202-206.

27. Chen BA, Jin N, Wang J, et al. The effect of magnetic nanoparticles of $\mathrm{Fe}_{3} \mathrm{O}_{4}$ on immune function in normal ICR mice. Int $J$ Nanomedicine. 2010;5:593.

28. Kosynkin DV, Higginbotham AL, Sinitskii A, et al. Longitudinal unzipping of carbon nanotubes to form graphene nanoribbons. Nature. 2009;458(7240):872-876.

29. Brewer BG, Mitchell RA, Harandi A, Eaton JW. Embryonic vaccines against cancer: An early history. Exp Mol Pathol. 2009;86(3): 192-197.

30. Grivennikov SI, Greten FR, Karin M. Immunity, inflammation, and cancer. Cell. 2010;140(6):883-899.

31. Liu Z, Cai W, He L, et al. In vivo biodistribution and highly efficient tumour targeting of carbon nanotubes in mice. Nat Nanotechnol. 2007; 2(1):47-52.

32. Poland CA, Duffin R, Kinloch I, et al. Carbon nanotubes introduced into the abdominal cavity of mice show asbestos-like pathogenicity in a pilot study. Nat Nanotechnol. 2008;3(7):423-428.

33. Magrez A, Kasas S, Salicio V, et al. Cellular toxicity of carbon-based nanomaterials. Nano Lett. 2006;6(6):1121-1125.

34. Zwaveling S, Ferreira Mota SC, Nouta J, et al. Established human papillomavirus type 16-expressing tumors are effectively eradicated following vaccination with long peptides. J Immunol. 2002;169(1):350-358.

35. Klebanoff CA, Gattinoni L, Restifo NP. CD8 T cell memory in tumor immunology and immunotherapy. Immunol Rev. 2006;211(1): 214-224.

36. Antony PA, Piccirillo CA, Akpinarli A, et al. CD8+ T cell immunity against a tumor/self-antigen is augmented by $\mathrm{CD} 4+\mathrm{T}$ helper cells and hindered by naturally occurring T regulatory cells. J Immunol. 2005; 174(5):2591-2601.

37. Janssen EM, Lemmens EE, Wolfe T, Christen U, von Herrath MG, Schoenberger SP. CD4+ T cells are required for secondary expansion and memory in CD8+ T lymphocytes. Nature. 2003;421(6925): $852-856$.

38. Dudley ME, Wunderlich JR, Robbins PF, et al. Cancer regression and autoimmunity in patients after clonal repopulation with antitumor lymphocytes. Science. 2002;298(5594):850-854.

39. Woll PS, Grzywacz B, Tian X, et al. Human embryonic stem cells differentiate into a homogeneous population of natural killer cells with potent in vivo antitumor activity. Blood. 2009;113(24):6094-6101.

40. Watarai H, Fujii S, Yamada D, et al. Murine induced pluripotent stem cells can be derived from and differentiate into natural killer T cells. J Clin Invest. 2010;120(7):2610-2618.

41. DeNardo DG, Andreu P, Coussens LM. Interactions between lymphocytes and myeloid cells regulate pro-versus anti-tumor immunity. Cancer Metastasis Rev. 2010;29(2):309-316.

42. Nagai H, Miyaki D, Matsui T, et al. Th1/Th2 balance: An important indicator of efficacy for intra-arterial chemotherapy. Cancer Chemother Pharmacol. 2008;62(6):959-963.

43. Clerici M, Clerici E, Shearer GM. Cytokine dysregulation in invasive cervical carcinoma and other human neoplasias: Time to consider the TH1/TH2 paradigm. J Natl Cancer Inst. 1998;90(4):261-263. 
44. Yamamoto M, Kamigaki T, Yamashita K, et al. Enhancement of anti-tumor immunity by high levels of Th1 and Th17 with a combination of dendritic cell fusion hybrids and regulatory $\mathrm{T}$ cell depletion in pancreatic cancer. Oncol Rep. 2009;22(2):337-343.

45. Apte $\mathrm{SH}$, Groves $\mathrm{P}$, Olver S, et al. IFN- $\gamma$ inhibits IL-4-induced type 2 cytokine expression by CD8 T cells in vivo and modulates the anti-tumor response. J Immunol. 2010;185(2):998-1004.

46. Ikeda $\mathrm{H}$, Chamoto $\mathrm{K}$, Tsuji $\mathrm{T}$, et al. The critical role of type-1 innate and acquired immunity in tumor immunotherapy. Cancer Sci. 2004; 95(9):697-703.

47. Shurin MR, Lu L, Kalinski P, Stewart-Akers AM, Lotze MT. Th1/ Th2 balance in cancer, transplantation and pregnancy. Springer Semin Immunopathol. 1999;21(3):339-359.

48. Keibel A, Singh V, Sharma MC. Inflammation, microenvironment, and the immune system in cancer progression. Curr Pharm Des. 2009; 15(17):1949-1955.

49. Dougan M, Dranoff G. Immune therapy for cancer. Annu Rev Immunol. 2009;27:83-117.

50. Calzascia T, Pellegrini M, Hall H, et al. TNF-alpha is critical for antitumor but not antiviral T cell immunity in mice. J Clin Invest. 2007; 117(12):3833.
51. Mocellin S, Rossi CR, Pilati P, Nitti D. Tumor necrosis factor, cancer and anticancer therapy. Cytokine Growth Factor Rev. 2005;16(1):35-53.

52. Heink S, Ludwig D, Kloetzel PM, Krüger E. IFN- $\gamma$-induced immune adaptation of the proteasome system is an accelerated and transient response. Proc Natl Acad Sci U S A. 2005;102(26):9241-9246.

53. Whitmire JK, Eam B, Benning N, Whitton JL. Direct interferon- $\gamma$ signaling dramatically enhances CD4 and CD8 T cell memory. J Immunol. 2007;179(2):1190-1197.

54. Loo DT. In situ detection of apoptosis by the TUNEL assay: An overview of techniques. Methods Mol Biol. 2011;682:3-13.

55. Zhao D, Alizadeh D, Zhang L, et al. Carbon nanotubes enhance CpG uptake and potentiate antiglioma immunity. Clin Cancer Res. 2011; 17(4):771-782.

56. Fadel TR, Look M, Staffier PA, Haller GL, Pfefferle LD, Fahmy TM. Clustering of stimuli on single-walled carbon nanotube bundles enhances cellular activation. Langmuir. 2010;26(8):5645-5654.
International Journal of Nanomedicine

\section{Publish your work in this journal}

The International Journal of Nanomedicine is an international, peerreviewed journal focusing on the application of nanotechnology in diagnostics, therapeutics, and drug delivery systems throughout the biomedical field. This journal is indexed on PubMed Central, MedLine, CAS, SciSearch ${ }^{\circledR}$, Current Contents ${ }^{\circledR} /$ Clinical Medicine,

\section{Dovepress}

Journal Citation Reports/Science Edition, EMBase, Scopus and the Elsevier Bibliographic databases. The manuscript management system is completely online and includes a very quick and fair peer-review system, which is all easy to use. Visit http://www.dovepress.com/ testimonials.php to read real quotes from published authors. 